\title{
De-noising $\boldsymbol{h}_{\text {int }}$ Surfaces: A Physics-Based Approach
}

\author{
Margaret Yam, Ralph Highnam, and Michael Brady \\ Medical Vision Laboratory, Engineering Science \\ Oxford University, Oxford OX1 3PJ, UK \\ \{margaret, rph, jmb\} @robots.ox.ac.uk
}

\begin{abstract}
The $h_{\text {int }}$ representation is a normalised, quantitative version of a mammogram which has substantial quantum noise components because of the way in which it is computed. This paper presents a physics-based approach to de-noising the $h_{\text {int }}$ representation of a mammogram. We investigate the major contributions to noise and the steps in the $h_{\text {int }}$ generation that amplify noise, such as removal of intensifying screen glare. Estimating the radiographic noise components using parameters derived from physics models, we filter the original mammographic images with an adaptive wiener filter, $W$. Generating the $h_{\text {int }}$ representation from the filtered images yields a de-noised version which has substantially improved signal-to-noise ratio, and which is far better to use for further-processing, such as microcalcification detection. The accuracy of the de-noised $h_{\text {int }}$ representation is verified using experimental results on phantom images and mammograms with microcalcifications.
\end{abstract}

Keywords: mammography, radiographic mottle, de-noising, physicsbased

\section{Introduction}

X-ray mammography plays an important role, and remains the primary imaging modality, in breast cancer screening. However, the quality of mammograms is always subject to variations in the imaging conditions; this makes robust and reliable mammographic analysis difficult, for human observers and machines alike. Acknowledging this fact, Highnam and Brady [3,4] developed a normalised mammographic representation, the $h_{\text {int }}$ representation, by modeling the X-ray imaging process and removing a number of image degrading factors, including scattered radiation, extra-focal radiation and intensifying screen glare. Every pixel value in the original image is converted into a floating point number which quantifies the amount of "interesting" (or non-fat) tissue present in the cone of breast above that pixel. However, removal of blur greatly amplifies the effects of high frequency noise componenets such as those due to radiographic mottle. Figure 3(a) and 4(a) show examples of noisy $h_{\text {int }}$ surfaces. The reduced signalto-noise ratio (SNR) of the noise-corrupted $h_{\text {int }}$ surface greatly hinders potential applications of it. The work presented in this paper is aimed at de-noising the $h_{\text {int }}$ 
surfaces by taking radiographic mottle into account. The correctness of the denoised $h_{\text {int }}$ surface is verified experimentally using both phantom images and mammograms with microcalcifications.

\section{Image Degrading Factors in the Mammographic Process}

Various image degrading factors exist in different parts of the mammographic imaging process. Some of the most important ones are:

- scattered radiation in the X-ray path through the breast;

- glare from the intensifying screen to the film; and

- radiographic mottle from the stochastic nature of the imaging process.

Scatter and glare result in low-frequency "noise" components and have been taken into account in the current $h_{\text {int }}$ model [3]. In this paper, we deal with the effect of radiographic mottle which introduces a high-frequency noise component. Some of the image degrading sources are depicted schematically in Figure 1.

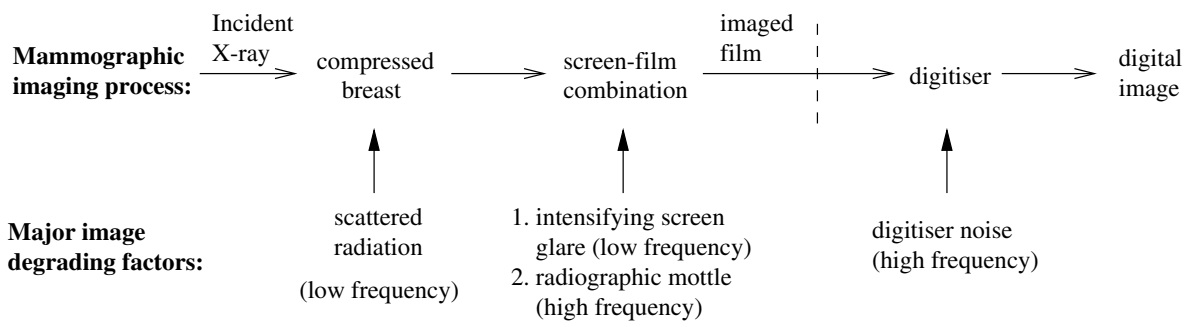

Fig. 1. A schematic representation of some of the major image degrading factors along the chain of mammographic imaging process.

Radiographic mottle is the spatial fluctuation of film density due to noise intrinsic to the screen-film combination, and comprises three primary components, namely quantum mottle, film granularity and structure mottle [1]. Quantum mottle $\left(\sigma_{q}(D)\right)$ is caused by the spatial fluctuations in the number of X-ray photons absorbed per unit area of the intensifying screen; film granularity $\left(\sigma_{g}(D)\right)$ is due to fluctuations in the number of silver halide grains per unit area of the film emulsion; and structure mottle $\left(\sigma_{s}(D)\right)$ arises from random inhomogeneities in the phosphor coating of the intensifying screen. Since these three components are independent random processes, the total radiographic mottle, $\sigma(D)$, can be formulated as:

$$
\sigma(D)=\left(\sigma_{q}^{2}(D)+\sigma_{g}^{2}(D)+\sigma_{s}^{2}(D)\right)^{\frac{1}{2}}
$$

where $\sigma(D)$ is the standard deviation of the film density, $D$. Amongst these three components, quantum mottle and film granularity contribute more significantly to the total radiographic noise while structure mottle makes up only $10 \%$ of it 
typically [7]. It is therefore reasonable to ignore for the present work the effect of structure mottle and approximate $\sigma(D)$ as:

$$
\sigma(D)=\left(\sigma_{q}^{2}(D)+\sigma_{g}^{2}(D)\right)^{\frac{1}{2}}
$$

Incorporating fluctuations in the energy absorbed per interacting photon and those in the number of light photons emitted per unit energy absorbed, Barnes [1] formulates $\sigma_{q}(D)$ as:

$$
\sigma_{q}(D)=\frac{0.434 G}{\left(n_{x} A_{e}\right)^{\frac{1}{2}}}\left[1+\frac{\left\langle\Delta E^{2}\right\rangle}{\langle E\rangle^{2}}\right]^{\frac{1}{2}}\left[1+\frac{\left\langle\Delta w^{2}\right\rangle}{\langle w\rangle^{2}}\right]^{\frac{1}{2}}
$$

where $G$ is the gradient of the film screen curve at density $D ; n_{x}$ is the average Xray photon fluence absorbed in the intensifying screen; $A_{e}$ is the effective noise sampling aperture; $\langle E\rangle$ and $\left\langle\Delta E^{2}\right\rangle$ are the mean and variance of the energy imparted in the screen per interacting photon; $\langle w\rangle$ and $\left\langle\Delta w^{2}\right\rangle$ are the mean and variance of the light photon yield per absorbed energy.

Film density fluctuation due to film granularity $\left(\sigma_{g}(D)\right)$ is given by:

$$
\sigma_{g}(D)=\left(\frac{0.434 a_{g}}{A}\right)^{2}[\langle D\rangle]^{\frac{1}{3}}
$$

where $a_{g}$ is the average grain area, $A$ the sampling area, and $\langle D\rangle$ is the average film density.

Both fluctuations in the number of absorbed X-ray photons and those in the number of film grains follow a Poisson distribution, but can be effectively modeled as Gaussian noise [2].

\section{The De-noising Algorithm}

The essence of our de-noising algorithm is to first filter radiographic mottle from the film density surface of the original mammogram and then generate the $h_{\text {int }}$ representation using the smoothed film density surface. We identify that the pronounced noise resulting in the $h_{\text {int }}$ representation, generated from intensity images, is due to amplification of the effect of quantum noise after removing the effects of blur due to glare from the intensifying screen. Glare arises from the isotropic emission of light by the phosphor in the intensifying screen to the film, which results in a small blurred exposure to the film. Its removal, therefore, increases image sharpness, but at the same time amplifies high frequency noise due to radiographic mottle. This is evident from the fact that the SNR of $h_{\text {int }}$ surfaces with glare removed is significantly smaller than those without glare removed (Table 2).

From Equations 3 and 4, it is obvious that the amplitudes of the noise components are signal-dependent. In the case of quantum mottle, $\sigma_{q}(D)$ is dependent on the photon fluence, $n_{x}$, which is in turn related to the film density via the film screen curve. For film grain noise, its standard deviation, $\sigma_{g}(D)$, is proportional 
to the cube root of the local average density. Modeling radiographic noise as being Gaussian distributed (which will be justified experimentally in Section 4.1), we filter the original film density surface using an adaptive wiener filter, $W$, [5], which is a spatially-variant filter taking local statistics into consideration. Given the original noisy signal $h$, elements of the filtered signal, $f(x, y)$, is:

$$
f(x, y)=m_{s}(x, y)+\frac{\sigma_{s}^{2}(x, y)}{\sigma_{s}^{2}(x, y)+\sigma_{n}^{2}(x, y)}\left(h(x, y)-m_{s}(x, y)\right)
$$

where $m_{s}(x, y)$ and $\sigma_{s}^{2}(x, y)$ are the signal mean and variance of a local neighbourhood around $(x, y)$ respectively; and $\sigma_{n}^{2}(x, y)$ is the local noise estimate computed using Equations 2, 3 and 4.

The de-noised $h_{\text {int }}$ surface is then obtained by generating the $h_{\text {int }}$ representation using methods described in [3] but having the original film densities replaced by the filtered version.

\section{Experimental Results}

\subsection{Radiographic Noise Analysis Using a Step Wedge Phantom}

We investigate the statistical properties of the radiographic noise using a 12-step lucite wedge phantom image. Experiments are performed on the middle 5 steps, the film densities of which lie in the linear region of the film-screen curve and correspond to the film density range within the breast area in mammograms. From the film density image of each step, we extract 24 smaller squares, each of $32 \times 32$ pixels, pair them off and obtain 12 difference images of film density by subtracting each image pair. The mean, variance, skew and kurtosis are computed for each difference image of each step. The plots are shown in Figure 2 and the average values for each step are presented in Table 1 . The skew and kurtosis values shown in Table 1 reflect that it is reasonable to approximate its distribution as Gaussian which has skew and kurtosis of 0 and 3 respectively.
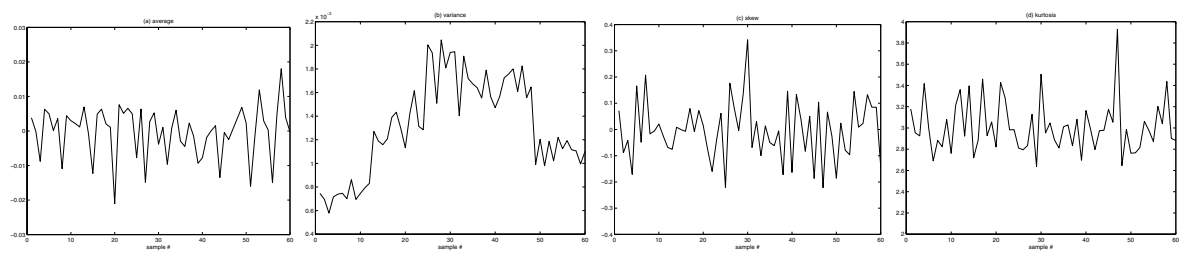

Fig. 2. Plots of (a) average, (b) variance, (c) skew, and (d) kurtosis of the difference images of film density. The x-axis refers to the label of individual samples. The line plots are solely for clearer presentation; no connectivity between records is implied. 


\begin{tabular}{|c|c|cccc|}
\hline step & average & \multicolumn{4}{|c|}{ difference image } \\
\cline { 3 - 6 } number & film density & average variance & skew & kurtosis \\
\hline \hline 4 & 0.54 & 0.0008 & 0.0007 & 0.0001 & 3.0235 \\
5 & 0.83 & 0.0010 & 0.0013 & -0.0109 & 3.0720 \\
6 & 1.30 & -0.0018 & 0.0018 & 0.0228 & 2.9541 \\
7 & 1.76 & -0.0024 & 0.0017 & -0.0243 & 3.0265 \\
8 & 2.19 & 0.0015 & 0.0011 & -0.0020 & 2.9753 \\
\hline
\end{tabular}

Table 1. Statistics of radiographic noise for different steps of the step wedge phantom

\subsection{De-noising $h_{\text {int }}$ Surfaces of Step Wedge Images}

We run our de-noising algorithm on the same 5 steps of the wedge as used in section 4.1. The images are digitised to a resolution of $50 \mu \mathrm{m}$ per pixel. Film-screen gradient $G$ is set to 3.6, corresponding to the gradient of the linear region of our film-screen curve. Sampling area, $A$, is set to the pixel area, which is $0.0025 \mathrm{~mm}^{2}$. We adopt the values of a couple of physical parameters as quoted in [1] $\left(A_{e}=0.79 \mathrm{~mm}^{2} ; a_{g}=5.31 \mu \mathrm{m}^{2}\right)$. Assuming the variance of energy absorbed per interacting photon, $\Delta E^{2}$, and that of light photon yield per absorbed energy, $\Delta w^{2}$, to be negligible, $\left[1+\left\langle\Delta E^{2}\right\rangle /\langle E\rangle^{2}\right]^{1 / 2} \approx 1$ and $\left[1+\left\langle\Delta w^{2}\right\rangle /\langle w\rangle^{2}\right]^{1 / 2} \approx 1 . n_{x}$ is computed from the energy imparted values. A mask size of $5 \times 5$ pixels is used to define the local neighbourhood in the filtering.

A comparison of the average, variance and signal-to-noise ratio (SNR) of the original $h_{\text {int }}$, the $h_{\text {int }}$ plus glare (i.e. the $h_{\text {int }}$ surface generated without glare removed), and the de-noised $h_{i n t}$ is given in Table 2. Since the $h_{\text {int }}$ surface of the step wedge should have constant height over each individual step, we define its SNR to be $\frac{\mu\left(h_{i n t}\right)}{\sigma\left(h_{i n t}\right)}$, where $\mu\left(h_{i n t}\right)$ and $\sigma\left(h_{i n t}\right)$ are the mean and standard deviation of $h_{\text {int }}$ values.

\begin{tabular}{|c|c|c|c|c|c|c|c|c|c|c|}
\hline \multirow[t]{2}{*}{ step } & \multirow{2}{*}{$\begin{array}{c}\text { lucite } \\
\text { thickness }\end{array}$} & \multicolumn{3}{|c|}{ original $h_{\text {int }}$} & \multicolumn{3}{|c|}{$h_{\text {int }}$ plus glare } & \multicolumn{3}{|c|}{ de-noised $h_{\text {int }}$} \\
\hline & & ave & var & SNR & ave & var & SNR & ave & var & SNR \\
\hline 4 & 4.40 & 2.15 & 0.168 & 5.23 & 2.11 & 10.003 & 37.8 & 2.11 & & 28 \\
\hline 5 & 3.96 & 2.02 & 0.143 & 5.34 & 1.99 & 0.00296 & 36.7 & 1.99 & 0.00512 & 27.8 \\
\hline 6 & 3.52 & 1.81 & 0.121 & 5.20 & 1.79 & 0.00238 & 36.7 & 1.79 & 0.00437 & 27.0 \\
\hline 7 & 3.08 & 1.69 & 0.092 & 5.58 & 1.68 & 0.00142 & 44.6 & 1.67 & 0.00261 & 32.7 \\
\hline 8 & 2.64 & 1.57 & 0.081 & 5.51 & 1.56 & 0.00128 & 43.5 & 1.55 & 0.00254 & 30.7 \\
\hline
\end{tabular}

Table 2. Comparison of average (ave), variance (var) and SNR of the original $h_{\text {int }}, h_{\text {int }}$ plus glare and de-noised $h_{\text {int }}$ surfaces of different steps of the step wedge phantom. 
It is interesting to note, from Table 2, that the average $h_{\text {int }}$ for the three versions of $h_{\text {int }}$ surface are very similar and are about $50 \%$ of the total lucite thickness, which agrees well to the fact that lucite approximates 50/50 interesting tissue/fat [3]. The increasing trend of variance with increasing lucite thickness of the steps reflects that as the thickness increases, the number of X-ray photons incident on the screen reduces hence increasing quantum noise.

The SNR of the de-noised $h_{\text {int }}$ surface is above 5 times larger than that of the original $h_{\text {int }}$, but is about 1.4 times less than that of the $h_{\text {int }}$ plus glare. This shows that the de-noised $h_{\text {int }}$ suppresses the noise power by a significant amount as compared to the original one. However, due to approximations made in the radiographic noise estimation, such as the omission of structure mottle, the SNR of the de-noised $h_{i n t}$ is slightly smaller than that of $h_{i n t}$ plus glare. The highest SNR of the $h_{\text {int }}$ plus glare also highlights the smoothing effect of glare on the $h_{\text {int }}$ surface. In the next section, we show that this blurring effect imposed by glare decreases image sharpness and affects the accuracy of the $h_{\text {int }}$ values.

Figure 3 shows the surface plots of the original $h_{\text {int }}$, the $h_{\text {int }}$ plus glare and the de-noised $h_{i n t}$ of one of the steps of the wedge, and their 1-D profiles along $y=100$.

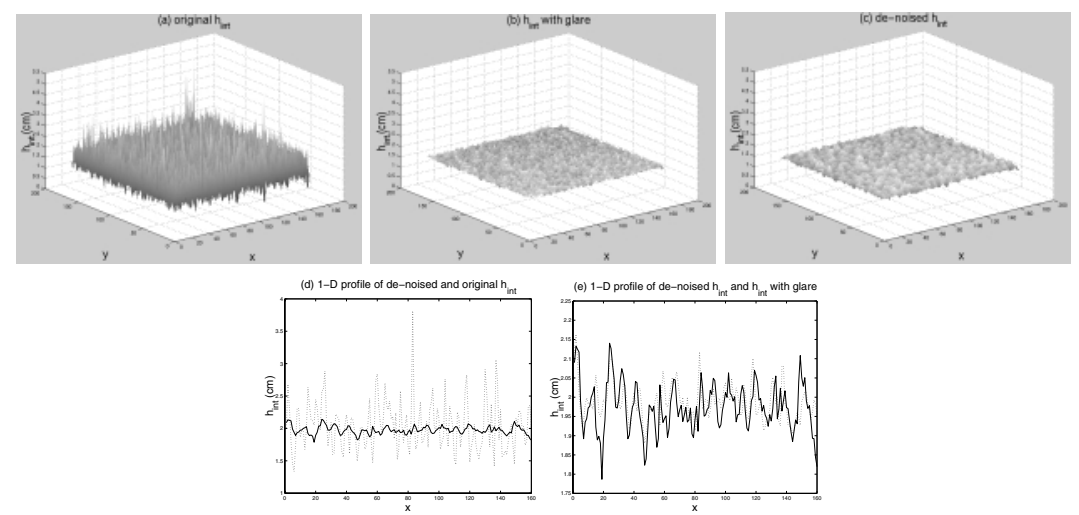

Fig. 3. (a)-(c): surface plots of the original $h_{\text {int }}$, the $h_{\text {int }}$ plus glare, and the de-noised $h_{\text {int }}$ of a step of the step wedge; (d): comparison of the 1-D profile of the original $h_{\text {int }}$ (dotted line) and the de-noised $h_{\text {int }}$ (solid line) along $y=100$; (e): comparison of the 1-D profile of the $h_{\text {int }}$ plus glare (dotted line) and the de-noised $h_{\text {int }}$ (solid line) along $y=100$.

\subsection{De-noising $h_{\text {int }}$ Surfaces of Microcalcification Images}

The experiment is performed on a set of 20 isolated microcalcifcations in 15 image samples, each of $230 \times 180$ pixels. All image samples are digitised to a resolution 
of $50 \mu \mathrm{m}$ per pixel. The de-noising procedure and parameters used are identical to that on the step wedge phantom given in Section 4.2. Figure 4(a)-(c) show the surface plots of the original $h_{\text {int }}$, the $h_{\text {int }}$ plus glare and the de-noised $h_{\text {int }}$ of one of the image samples with a microcalcifications about (120, 74). Figure 4(d) and (e) compare the 1 -D profiles, across the microcalcification at $y=74$, of the de-noised $h_{\text {int }}$ with that of the original $h_{\text {int }}$ and the $h_{\text {int }}$ plus glare respectively. It can be seen from the plots that whilst removing much of the noise from the original $h_{\text {int }}$ surface, the de-noised $h_{\text {int }}$ retains image contrast and sharpness without inducing a blurring effect on the microcalcification as in the case of the $h_{\text {int }}$ plus glare.

Using a physics-based model [3], we simulate the X-ray mammographic imaging process and obtain simulated image samples from the original $h_{\text {int }}$, the $h_{\text {int }}$ plus glare and the de-noised $h_{\text {int }}$ representation. Figure 5 show their respective appearances along with the original mammogram image sample. The better image quality of the de-noised $h_{\text {int }}$ over the original $h_{\text {int }}$, and the improved image contrast and sharpness of the de-noised version over the one with glare and the original image are apparent.

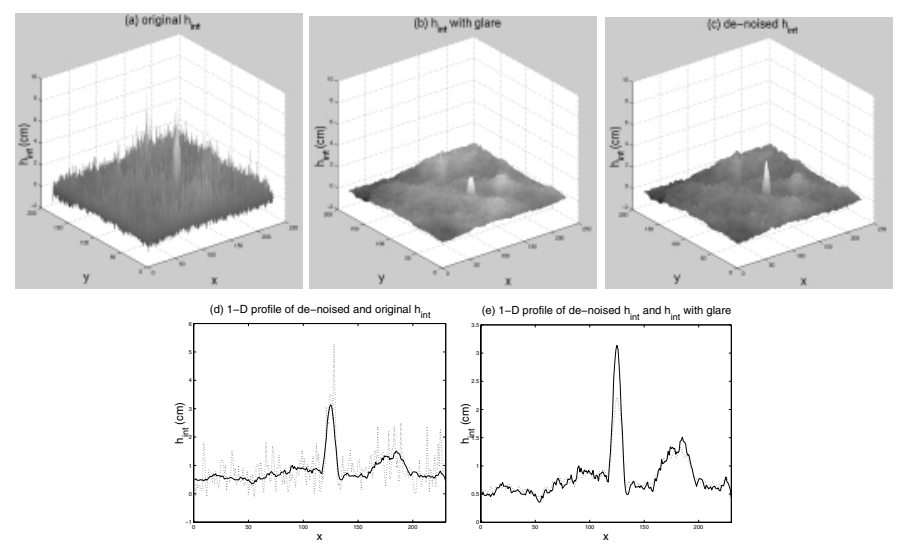

Fig. 4. (a)-(c): surface plots of the original $h_{\text {int }}$, the $h_{\text {int }}$ plus glare, and the de-noised $h_{\text {int }}$ of a mammogram sample with a microcalfication about $(120,74)$; (d): comparison of the 1-D profile of the original $h_{\text {int }}$ (dotted line) and the denosied $h_{\text {int }}$ (solid line) along $y=74$; (e): comparison of the 1-D profile of the $h_{\text {int }}$ plus glare (dotted line) and the de-noised $h_{\text {int }}$ (solid line) along $y=74$.

To evaluate the accuracy of the de-noised $h_{\text {int }}$ model, we use it to quantify microcalcifications by means of their volume ratios to their corresponding estimated 3-D volumes based on an ellipsoid model. The method is similar to that described in [8]. The segmentation uses iso-contours and an area constraint on the de-noised $h_{\text {int }}$ surface to pick out regions which correspond to candidate 
(a)

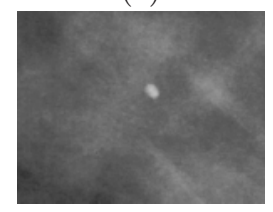

(b)

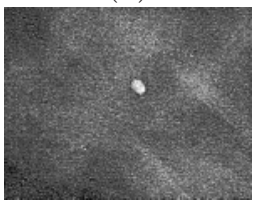

(c)

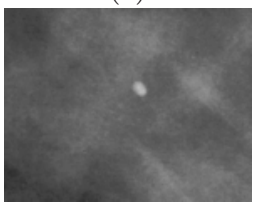

(d)

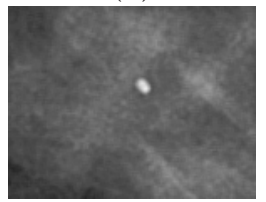

Fig. 5. (a): original mammogram sample; simulated X-ray mammograms using (b): the original $h_{\text {int }}$, (c): the $h_{\text {int }}$ plus glare, and (d): the de-noised $h_{i n t}$ respectively.

calcifications. The actual calcification thickness, $h_{\text {calc }}$, is related to the $h_{\text {int }}$ values by (from [3]): $h_{\text {calc }}(x, y) \approx 0.018 \times\left(h_{\text {int }}(x, y)-h_{\text {int }}^{\text {surr }}\right)$, where $h_{\text {int }}^{\text {surr }}$ is the average $h_{\text {int }}$ thickness of the surrounding tissue obtained by performing a morphological dilation of the extracted region. Then we compute the ratio of the microcalcification volume to its estimated 3 -D volume (the computation of both quantities is described in [8]), $v_{\text {calc }}^{\text {ratio }}$, which has a theoretical value of 1 .

The computed $v_{\text {calc }}^{\text {ratio }}$ s of the 20 microcalcifications, using the three versions of $h_{\text {int }}$, are given in figure 6(a). The values obtained using the de-noised $h_{\text {int }}$ approximate the theoretical value of 1 , while those using the original $h_{\text {int }}$ are greater than 1 due to the inclusion of quantum noise and those using $h_{\text {int }}$ with glare are less than 1 due to the excessive smoothing that lowers the $h_{\text {int }}$ values. The rms error of the $v_{\text {calc }}^{\text {ratio }}$ from 1 is $0.062,0.511$ and 0.415 for the de-noised $h_{\text {int }}$, the original $h_{\text {int }}$ and the $h_{\text {int }}$ plus glare respectively.

This physical quantity, $v_{\text {calc }}^{\text {ratio }}$, can be used in a quantitative approach for microcalcification detection. Previous work using volume to characterise calcifications can be found in [6]. In an initial trial, the 20 microcalcifications are all successfully detected with no false positives using a $v_{\text {calc }}^{\text {ratio }}$ threshold of 0.8. Using Receiver Operating Characteristics (ROC) analysis, we show that our results are better than those obtained using a grey level contrast measure and thresholding (Figure 6(b)).

\section{Discussion and Conclusions}

We have presented a physics-based approach to de-noising $h_{\text {int }}$ surfaces. The reliability of our restoration scheme is related to the underlying physics model of radiographic mottle in terms of a number of physically-defined parameters, hence eliminating the need to fine-tune variables in a heuristic manner.

The accuracy of our de-noised $h_{\text {int }}$ model was verified experimentally using a step wedge phantom image and mammograms with microcalcifciations. The computed $h_{\text {int }}$ thickness of steps of the phantom approximates the theoretical value, which is half of the lucite thickness of the corresponding step. On microcalcifications, we quantify them by means of the physical quantity, $v_{\text {calc }}^{\text {ratio }}$, which 


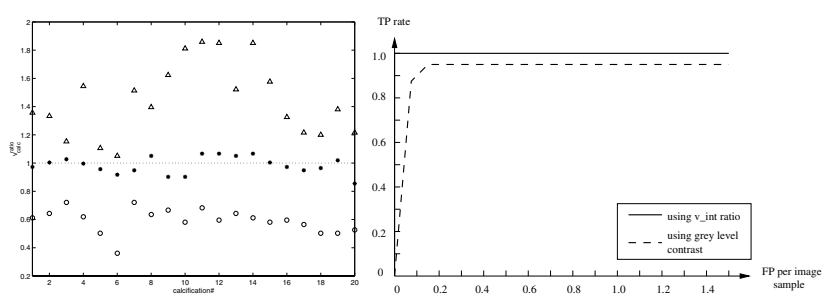

Fig. 6. Left: A scatter plot showing the computed $v_{\text {calc }}^{\text {ratio }}$ of 20 microcalcifications using the original $h_{\text {int }}$ (triangle), the $h_{\text {int }}$ plus glare (circle), and the denoised $h_{\text {int }}$ (star); Right: Comparison of microcalcification detection results using our method and using grey level contrast thresholding.

is the ratio of the computed volume of a microcalcification to its estimated 3$\mathrm{D}$ volume. We have shown that $v_{\text {calc }}^{\text {ratio }} \mathrm{s}$ computed on the de-noised $h_{\text {int }}$ surface have smallest deviations from the theoretical value of 1 as compared with those obtained from the original $h_{\text {int }}$ and the $h_{\text {int }}$ plus glare.

In essence, our de-noising algorithm is capable of rescuing the $h_{\text {int }}$ from the amplified effect of quantum noise without inducing a blurring effect on the $h_{\text {int }}$ signal. The de-noising algorithm also makes feasible applications concerning analysis of the $h_{\text {int }}$ surface, such as the detection of calcifications by means of quantitative volume analysis. Future work will employ the de-noised $h_{\text {int }}$ representation on microcalcification detection and evaluate its performance using a larger data set.

\section{Acknowledgements}

Margaret Yam is supported by a postgraduate scholarship from the Croucher Foundation (Hong Kong) and an Overseas Research Student Award (UK). Ralph Highnam and Michael Brady's work in mammography is supported by a grant from the Engineering and Physical Sciences Research Council (UK).

\section{References}

1. G. T. Barnes. Radiographic mottle: A comprehenive theory. Medical Physics, 9(5):656-667, 1982. 228, 229, 231

2. K. R. Castleman. Digital Image Processing, Prentice Hall, 1996. 229

3. R. P. Highnam, and J. M. Brady. Mammographic Image Processing, Kluwer Academic Publishing, 1999. 227, 228, 230, 232, 233, 234

4. R. P. Highnam, M. Brady, and B. J. Shepstone. A representation for mammographic image processing. Medical Image Analysis, 1:1-19, 1996. 227

5. J. S. Lee Digital image enhancement and noise filtering by use of local statistics. IEEE Transaction on Pattern Analysis and Machine Intelligence, 2:165-168, 1980. 230 
6. R. M. Nishikawa, Y. Jiang, M. L. Giger, C. L. Vyborny, R. A. Schmidt, and U. Bick. Characterization of the mammographic appearance of microcalcifications: applications in computer-aided diagnosis. SPIE vol.1898 Imag. Proc. 422-29, 1993. 234

7. S. Webb. The Physics of Medical Imaging, Institute of Physics Publishing, 1988. 229

8. M. Yam, J. M. Brady, R. P. Highnam, and R. English. Detecting calcifications using the $h_{\text {int }}$ representation. The 13th International Congress on Computer Assisted Radiology and Surgery, Paris, June 1999. 233, 234 